\title{
Recipe Recommendation
}

\author{
Soundarya Desai ${ }^{1}$, Pooja Patil ${ }^{2}$, Pratik Shinde ${ }^{3}$, Azhar Sayyed ${ }^{4}$, Prof. Rohini Bhosale ${ }^{5}$ \\ Student, Comp Dept, KJEI'S Trinity College of Engineering and Research, Pune, India ${ }^{1,2,3,4}$ \\ Guide, Comp Dept, KJEI'S Trinity College of Engineering and Research, Pune, India ${ }^{5}$
}

\begin{abstract}
In this paper, we propose recipe recommendation system that using a data. With the power of web, aggregate world is related and particular customers of different countries are sharing the number of recipe on the Net. Along these lines, thusly customers don't think about the each one of the recipe on the web. This paper presents a recipe recommendation system to recommend a set of dishes from the various Chinese regional cuisines for a certain flavor preference in terms of flavor similarity[1]. Recipe contains differing fixings, cooking strategy, classes so on. Thusly, we think the recipe is combination of the extraordinary heterogeneous components. Most of the proposal structure relies upon the substance or group situated filtering to predict the new recipe of eagerness for a customer. Joining with the both the filtering techniques, we display a fruitful and wonderful structure for uniting the two methodologies in Recipe proposal system. A substantial part of the equation proposition system uses content information as fixings or cooking procedures of Recipe. We proposed novel way to deal with prescribe new recipes. With life style, diet habits has changed and work pressure increased which resulted in number of diseases, such as diabetes, Blood Pressure, heart problem etc. These diseases can be controlled to certain extent by avoiding uneven and inappropriate diet.[2]
\end{abstract}

Keywords: Recommendation System, Collaborative Filtering, Recipes, Content Information

\section{INTRODUCTION}

In Many people often cook a dish with a cooking recipe on Websites and mgzines.[1]Recommendation System is known as a successful answer for data over-burden and there are numerous proposal inquire about accomplishments have been produced using the scholarly world and industry. By diming a binary connection between client item, recommendation framework can enable client to discover what they are interested in, and construct customized suggestion to address their issues. Late years, the term Kitchen economics matters has been ascended, and portable applications for Providing recipe data has steadily turned into the concentration in our life. In any case, the developing portable Internet administrations and Content will continuously surpass the worthy scope of individuals. In this paper, an adaptable recommendation framework are outlined and executed for customized recipe proposal for web applications and versatile apps. We are using JAVA and MYSQL to design the whole system interface (UI). Through the UI we can understand the user's preferences and his/her daily amount of nutrients that can be ingested. Based on the user preference the system can extract from the knowledge base in line with a number of sample recipes for recommendation. In this paper proposes another technique in view of machine learning. The formulas are produced in view of existing ones utilizing straight blends. In the event that the produced recipe can't pass its assessment isn't prescribed. Then again, if a formula can pass the assessment, we do prescribe it to clients.

\section{LITERATURE SURVEY}

Title:-"Recommendation System for Alternative-ingredients Based on Co-occurrence Relation on Recipe Database and the Ingredient Category."

Author:-Naoki SHINO, Rousse YAMANISHI, Junichi FUKUMOTO2016 5th IIAI International Congress on Advanced Applied Informatics.[1]

Many people often cook a dish with a cooking recipe on Websites and magazines. The listed ingredients in the recipe sometimes cannot be prepared. This paper proposes a recommendation system for alternative ingredients. The recommendation ingredients based on co-occurrence frequency of ingredients on recipe database and ingredient category stored in a cooking ontology. The result of the subjective evaluation experiments showed $88 \%$ appropriateness for alternative-ingredients recommendation.

Title:-“A scalable recipe recommendation system for mobile application."

Author: - ZhengXian Li, Jinlong Hu*, Jiazhao Shen, Yong Xu2016 3rd International Conference on Information Science and Control Engineering.[2] 


\section{International Journal of Advanced Research in Computer and Communication Engineering}

Vol. 7, Issue 11, November 2018

There are more and more users to use mobile application to get the cooking recipe information, but to recommend right recipe for each user is challenging for the complex food and diet information. In this paper, a hybrid recommendation system is proposed for personalized recipe mobile application. We describe a scalable recommendation system to process the massive data from mobile applications based on the spark clusters, and design a hybrid recommendation algorithm, which combines content-based filtering and collaborative filtering to improve the recipe recommended effectiveness. Our experiments reveal that the recommendation system has scalable computational capability.

Title :- "Distributed Representation of Dish Names in Food Related Web Services for Associative Search and Nutrition Estimation."

Author :- Yuguan Xing,Sosuke Amano Toshihiko Yamasaki ,Kiyoharu Aizawa, Makoto Ogawa2017 IEEE Third International Conference on Multimedia Big Data.[3]

Food related web services, such as recipe websites and food journaling apps, are rapidly gaining popularity. Data from service providers and that generated by users often coexist in these services. Compared to the former, the latter, due to its randomness and lack of organization, is often difficult to incorporate into common service features like recommendation making and associative searching. This work addresses this problem by investigating the effectiveness of multiple text embedding methods in generating distributed representations of dish titles that accurately capture the concepts of the dishes which these titles are referring to. We evaluated the practical potential of these "food vectors" with a retrieval task and a nutrition estimation task, and concluded that a method based on word embedding and LSTM is most suited for these purposes.

\section{Title :- "A Content-aware Fridge Based on RFID in Smart Home for Home-Healthcare." Author :- Hanshen Gu, Dong Wang School of Software, Shanghai Jiaotong University[4]}

With the ever increasing popularity of Radio Frequency Identification, it is easier for us to lead a more convenient and healthier life. In this paper we present a Content-aware Fridge based on RFID to provide several content-aware services to adjust our Eating habits. Especially, for patients, the system is able to inform them to take certain food which is good for their health and print a shopping list intelligently when the stock of fridge is in short. With the foodontology, we analyze the nutrients between the different types of food. And make recommendations on recipe so as to ensure that each of the family members will receive a well-balanced diet.

Title:- "Automatic Generation and Recommendation of Recipes Based on Outlier Analysis." Author :- Yu-Wen Lo, Qiangfu Zhao, Yu-Hsien Ting, and Rung-Ching Chen[5]

Research results on medicine and health show that people nowadays tend to have some common diseases because of abnormal eating habits, irregular lifestyles, fast-food culture, etc. Diabetes and high blood pressure are just two examples. This study is based on an ontology-based dietary management system Established by our group earlier. The main contribution of this paper is to propose a method for synthesizing new recipes based on existing ones, and recommending proper recipes based on machine learning. The new recipes are combinations of several existing ones. They are recommended to the user only if necessary nutrition's are properly contained in the recipe. Outlier Analysis is used to judge if a recipe is good or not. Some primary experiments are conducted to show the usefulness of the proposed method.

\section{PROPOSE SYSTEM}

In Proposed system we analysing the Android application .So, in that the user can register the application then registration is successful then login the application. As well as Admin can register the application then registration is successful then login application. After login User can upload recipe image. Then this image is display in Admin dashboard. Then admin after login View this image and substantiate the image. Then user can Add new recipe and also give rate as well as again view this recipe.

\section{SYSTEM ARCHITECTURE}

Following diagram is our system's architecture diagram:

In System architecture Admin and user can register the application and registration is successful then login the application. Then User upload image in Android application. Then this image is Sustained that is this image is Analysis and verified. Similarly in web application user can add the new recipe and rate to recipe. 


\section{International Journal of Advanced Research in Computer and Communication Engineering}

Vol. 7, Issue 11, November 2018

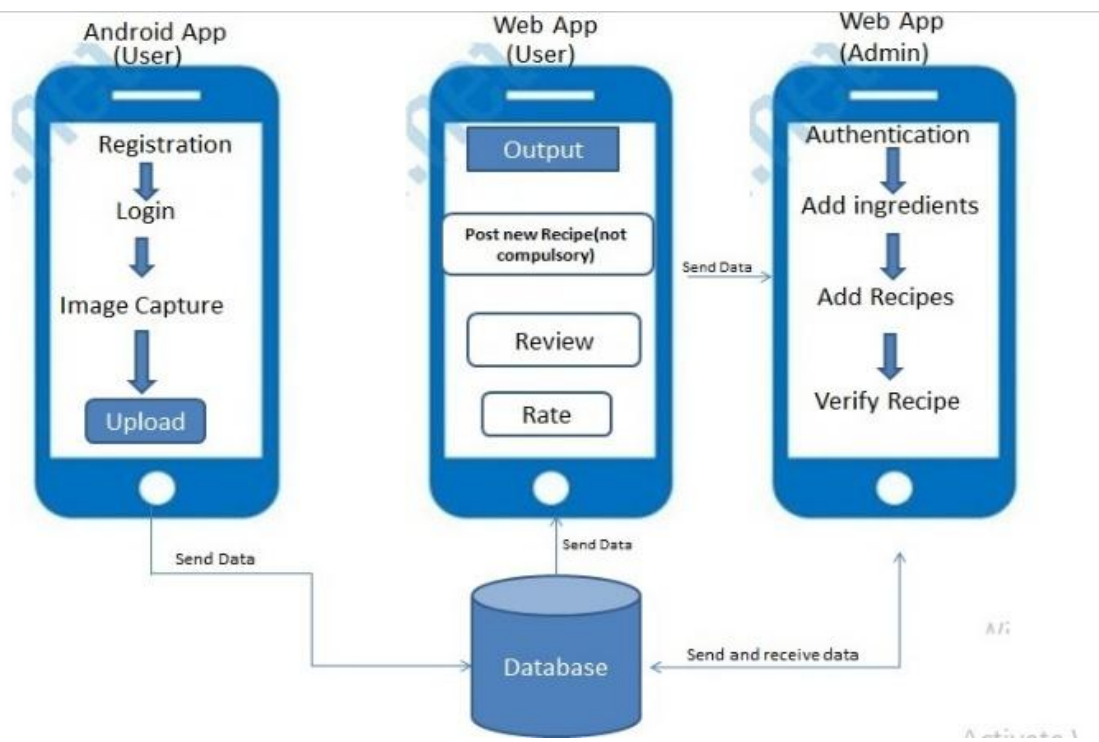

Figure 1: system architecture

\section{MODULES}

There system is divided into three parts according to functions performed by individuals. In this system we need to collect the data from different resources like recipes, ingredients, nutrition values for different recipes so that we are divide the system in two subsections:-

1. Admin:-Admin part manages all data related to recipe like add recipe delete recipe, manage ingredients. Admin need to perform the verification of recipes posted by the user with some basic user management functions.

2 .User:- User needs to authenticate with system and upload different images from application. The uploaded image will be displayed on webpage and system identifies the ingredients from the Image and suggest recipe according to ingredients.

\section{CONCLUSION}

Recommendation system is the mechanism of recommendation of recipe. The system reveals the detailed description of our system. Generally speaking, the consensus result could be achieved by iteratively refining the local training result. The system is being proposed for food lover people. It is very efficient than existing system. The system give efficient output of recommendation. The proposed system features a low computation cost and confidentiality of the training set

\section{REFERRENCES}

[1]. "Recommendation System for Alternative-ingredients Based on Co-occurrence Relation on Recipe Database and the Ingredient Category."Naoki SHINO Rousse YAMANISHI, Junichi FUKUMOTO2016 5th IIAI International Congress on Advanced Applied Informatics.

[2]. "Constraint Based Recipe Recommendation using Forward Checking Algorithm.” 2016 Intl. Conference on Advances in Computing, Communications and Informatics (ICACCI), Sept. 21-24, 2016, Jaipur, India Kirti.Pawar, TusharGhorpade, Rajashree Shedge

[3]. "A scalable recipe recommendation system for mobile application.” ZhengXian Li, Jinlong Hu*, Jiazhao Shen, Yong Xu2016 3rd International Conference on Information Science and Control Engineering.

[4]. "Distributed Representation of Dish Names in Food Related Web Services for Associative Search and Nutrition Estimation." Yuguan Xing,Sosuke Amano Toshihiko Yamasaki ,Kiyoharu Aizawa, Makoto Ogawa2017 IEEE Third International Conference on Multimedia Big Data.

[5]. "A Content-aware Fridge Based on RFID in Smart Home for Home-Healthcare."Hanshen Gu, Dong Wang School of Software, Shanghai Jiaotong University.

[6]. "Automatic Generation and Recommendation of Recipes Based on Outlier Analysis." Yu-Wen Lo, Qiangfu Zhao, Yu-Hsien Ting, and Rung-Ching Chen

[7]. P.K.Paul and M.K.Ghose, "Cloud computing: possibilities, challenges and opportunities with special reference to its emerging need in the academic and working area of Information Science," In Procedia Engineering, vol. (23), pp.2222-2227, Jan 2012.

[8]. Kandukuri, Balachandra Reddy, and Atanu Rakshit, “Cloud security issues,” InServices Computing, 2009. SCC\&\#39;09. IEEE International Conference on IEEE, pp. 517-520, Sep 2009.

[9]. L. Adleman, "Molecular computation of solutions to combinational problems".American Association for the Advancement of Science, pp.1021-1024, 1994.

[10]. Rachna.A, and Anshu.P, "Maintaining Data Confidentiality and Security over Cloud: An Overview", International Journal of Engineering Research and Applications (IJERA), Vol. (4), pp.1922-1926, July 2013.

[11]. D.Sureshraj and Dr.V.Murali Bhaskaran, Automatic DNA Sequence Generation for Secured Effective Multi-Cloud Storage, Journal of Computer Engineering (IOSR-JCE), vol.15, pp. 86-94, Nov-Dec 2013. 